Cross-section comparisons of cloaks designed by transformation optical and optical conformal mapping approaches

This article has been downloaded from IOPscience. Please scroll down to see the full text article.

2011 J. Opt. 13024002

(http://iopscience.iop.org/2040-8986/13/2/024002)

View the table of contents for this issue, or go to the journal homepage for more

Download details:

IP Address: 152.3.31.220

The article was downloaded on 16/11/2010 at 17:34

Please note that terms and conditions apply. 


\title{
Cross-section comparisons of cloaks designed by transformation optical and optical conformal mapping approaches
}

\author{
Yaroslav A Urzhumov ${ }^{1}$, Nathan B Kundtz ${ }^{1,2}$, David R Smith ${ }^{1}$ and \\ John B Pendry ${ }^{3}$ \\ ${ }^{1}$ Center for Metamaterials and Integrated Plasmonics, Pratt School of Engineering, \\ Duke University, Durham, NC 27708, USA \\ ${ }^{2}$ Intellectual Ventures, 3150 139th Ave SE Building, Bellevue, WA 98005, USA \\ ${ }^{3}$ The Blackett Laboratory, Imperial College London, London SW7 2AZ, UK \\ E-mail: yaroslav.urzhumov@duke.edu and j.pendry@imperial.ac.uk
}

Received 11 May 2010, accepted for publication 28 July 2010

Published 16 November 2010

Online at stacks.iop.org/JOpt/13/024002

\begin{abstract}
We review several approaches to optical invisibility designed using transformation optics (TO) and optical conformal mapping (CM) techniques. TO is a general framework for solving inverse scattering problems based on mimicking spatial coordinate transformations with distributions of material properties. There are two essential steps in the design of TO media: first, a coordinate transformation that achieves some desired functionality, resulting in a continuous spatial distribution of constitutive parameters that are generally anisotropic; and, second, the reduction of the derived continuous constitutive parameters to a metamaterial that serves as a stepwise approximation. We focus here on the first step, discussing the merits of various TO strategies proposed for the long-sought 'invisibility cloak' — a structure that renders opaque objects invisible. We also evaluate the cloaking capabilities of structures designed by the related CM approach, which makes use of conformal mapping to achieve index-only material distributions. The performance of the various cloaks is evaluated and compared using a universal measure- - the total (all-angle) scattering cross section.
\end{abstract}

Keywords: transformation optics, optical conformal mapping, cloak, scattering cross section, eikonal approximation, geometrical optics, metamaterials

(Some figures in this article are in colour only in the electronic version)

\section{Introduction}

Transformation optics (TO) is a recently appreciated tool for the design of complex media with unique wave propagation properties [1, 2]. Introduced in the present context as a computational technique to extend the utility of finitedifference [3] and finite-element codes [4], TO has become widely appreciated for its generality and ability to design structures that manipulate waves with unprecedented control. The main tool associated with TO is that of coordinate transformations, in which isotropic space is conceptually warped or otherwise distorted as a means of guiding the trajectories of waves. The coordinate transformation that results in some desired functionality can then be used to determine the properties of a physical medium in which waves will behave as if they were propagating in the warped space. The TO method can be applied to any linear waves for which the underlying equations exhibit form invariance under coordinate transformations. Maxwell's equations, for 
example, are generally form-invariant, so that coordinate transformations can equivalently be implemented as spatially varying, anisotropic constitutive parameters (i.e. the electric permittivity and the magnetic permeability) [5-8].

Coordinate transformations can be considered the primary tool of TO, in analogy to the use of ray tracing for geometrical optics (GO). However, the tools are quite different in many aspects, since TO is exact to the level of Maxwell's equations, while geometrical optics ignores the wave-like properties. Ray tracing allows one to determine the trajectory of rays incident on or through a medium; a medium or device that accomplishes some function can then be arrived at, for example, by optimization. In contrast, coordinate transformations allow one to start from the desired functionality and arrive immediately at the medium properties that will accomplish the goal. TO designs are not unique; not only can a TO solution always be found that achieves a desired function, usually an infinite set of transformations exist, each corresponding to a different hypothetical medium. The TO media that ensue from a transformation are generally anisotropic [8], spatially varying and possess both an electric as well as magnetic response. That is, the TO approach inherently assumes no constraints on the complexity of the material. Whether a material can actually be manufactured that corresponds to a given transformation is uncertain, and usually optimization and simplification steps would be needed to arrive at a feasible design.

The complexity of the materials needed to achieve TO designs will typically exceed what can be obtained from naturally occurring media. Fortunately, the last decade of research into artificially structured media-often termed metamaterials - has shown that a much wider range of material response is available through engineered composites [9-13]. Metamaterials have been constructed to have electromagnetic or acoustic responses that do not exist in nature, such as negative refractive index [9]. The promising results that have emerged from the metamaterials community have fueled the investigation of novel design approaches such as TO, since it is now possible to realize the actual specified media-even if only in a stepwise or other approximate manner.

The TO approach can be used to design exotic and unusual structures in an entirely intuitive process. Perhaps one of the most thought-provoking examples of a TO structure has been that of the invisibility cloak, a device that would prevent light or electromagnetic waves from scattering from an enclosed object. The transformation for a radially symmetric cloak is relatively simple and has been presented in numerous places: it compresses the space contained in some volume into a shell about that volume [1]. The continuous material resulting from such a transformation must seamlessly guide light around an enclosed object placed in the central concealment volume. An external observer would thus be unable to distinguish between the presence of the object (and the cloak), and empty space. A natural and appropriate description for such situations is to consider the object as 'cloaked', or invisible to an observer.

Real materials or metamaterials can only approximate the continuous, spatially varying material parameters required by the transformation. In the context of cloaking, any physical realization will involve materials that exhibit some degree of material losses, frequency dispersion, magnetoelectric coupling, discreteness or a myriad of other undesired properties. These factors will render the cloak imperfect, leading to some amount of scattering of the incident waves. In 2006, an approximate TO cloak constructed using metamaterial elements was demonstrated at microwave frequencies that manifested the expected traits of the TO design, but revealed considerable reflection and shadowing [14]. The amount of residual scattering provides a natural measure by which to quantify the success of an implementation of a cloaking transformation. Although a cloak is hardly the only type of device of interest via the TO approach, it lends itself particularly well to characterization and can be utilized as a means of quantifying successive improvements in TO implementations [15]. Alternatively, the efficacy of different cloaking transformations and their approximations can be evaluated by the degree of unwanted scattering.

The performance of a cloak can be conveniently quantified by its scattering cross section (SCS). The SCS is defined as the total energy in the scattered fields divided by the energy per unit area (per length in two dimensions) in the incident field, and can be thought of as an effective size associated with the scattering object. The SCS has units of area in three dimensions and length in two. The expected function of all cloaking transformations is to cause an object to appear smaller than its actual physical extent, as defined according to its SCS. A perfect cloak can be defined as a structure that shrinks the apparent size of an object to zero. The SCS is not a unique figure-of-merit for cloaking efficacy, but it is a reasonable quantity by which to compare various cloaking strategies.

Numerous proposals for implementations of cloaking have appeared in the literature over the past few years, in parallel with the overall development of TO structures. Given that there are advantages and difficulties associated with every method, it is useful to compare a variety of approaches using a common quantitative measure, such that trade-offs in performance versus potential ease in realization can be understood. In section 2 , we consider a variety of exact and approximate TO approaches to cloaking, using the SCS as the figure-of-merit. In section 3, we focus on cloaking via the related technique of optical conformal mapping (CM) [12]. The CM approach can be distinguished from the TO approach in that it is restricted to the geometrical optics limit, so that the full properties of a wave are not managed by the CM material. The CM approach is advantageous in that it requires media that can be formed from isotropic, inhomogeneous dielectric materials. However, such media can never provide complete cloaking, which would violate the uniqueness theorem [16-18]. The degree to which certain proposed CM designs can achieve cloaking is explored in section 3 .

\section{Transformation optics approach}

TO transformations are entirely general, being able to map nearly any volume with an arbitrary bounding surface into any other arbitrarily shaped volume. For convenience, we restrict our attention here to radially symmetric transformations, which 
typically are easily expressed as analytical expressions. In two dimensions, for example, a cylindrically symmetric perfect cloak can be derived for a coordinate transformation $r^{\prime}=q(r)$ that maps an annulus $a<r<b$ onto the region $0<$ $r^{\prime}<b$ [1, 14]. For such transformations, the constitutive parameters in the physical space (with radial coordinate $r$ ) corresponding to $\epsilon^{\prime}=\mu^{\prime}=1$ in the transformed spaceshould be chosen as follows $[14,19]$ :

$$
\begin{gathered}
\epsilon_{r}=\mu_{r}=(q / r) / q^{\prime}, \quad \epsilon_{\theta}=\mu_{\theta}=q^{\prime} /(q / r), \\
\epsilon_{z}=\mu_{z}=q^{\prime}(q / r),
\end{gathered}
$$

where $q^{\prime} \equiv \mathrm{d} q(r) / \mathrm{d} r$. Note that we are using the transformation that is inverse to what is used in some of the literature $[14,19]$.

For radial transformations in three dimensions, the following material parameters within the shell $a<r<b$ are needed to accomplish perfect invisibility $[1,20]$ :

$$
\begin{aligned}
& \epsilon_{r}=\mu_{r}=n_{\theta}^{2} / n_{r}=(q / r)^{2} / q^{\prime}, \\
& \epsilon_{\theta}=\mu_{\theta}=\epsilon_{\phi}=\mu_{\phi}=n_{r}=q^{\prime} .
\end{aligned}
$$

The transformation function $q(r)$ must obey the two boundary conditions: $q(a)=0$ ensures that the cloak interior is transformed to a point in $r^{\prime}$ space and $q(b)=b$ guarantees continuity of transformation, which must continue as $q(r)=r$ at $r>b$. The simplest, linear function satisfying these boundary conditions is $[1,14,21,22]$

$$
q(r)=b(r-a) /(b-a) .
$$

Imperfect cloaks with a less restrictive requirement $q(r=$ $a)=q_{\min } \ll a$ have also been proposed as a realistic approximation to the perfect cloak [23, 24].

To simulate the operation of cloaking structures and assess their performance, we make use of COMSOL Multiphysics, a commercial finite-element analysis package. The accuracy of COMSOL for such studies has been documented in prior works $[14,21]$. Throughout this paper, we neglect the effect of electromagnetic losses by assuming real-valued constitutive tensor elements; in addition, all material properties are assumed non-dispersive (frequencyindependent). Consequently, all calculations of this section can be scaled to arbitrary wavelengths by rescaling of the cloak dimensions. Reference [25] offers an interesting analysis of dispersive properties of cylindrical cloaks.

\subsection{Role of interior boundary of the cloak}

It is well known [26] that cloaks may allow partial field penetration into their interior cavity (the region $r<a$ ), mostly due to various imperfections of their implementation. Imperfections might include the finite size of metamaterial elements; the bounding of divergences in one or more of the constitutive tensor elements; and eikonal approximations (see section 2.4). In numerical simulations, the penetration of fields into the interior of the cloak-where they would otherwise undergo scattering - are blocked through the use of insulating boundary conditions frequently imposed at $r=a$. Two of the most common insulating conditions are the so-called hard (Dirichlet) and soft (Neumann) conditions. For instance, a perfect electric conductor (PEC) boundary can be inserted on the $r=a$ layer, which for transverse electric (TE) waves will serve as a hard insulating boundary $\left(E_{z}=0\right)$. A perfect magnetic conductor (PMC) on the inner layer will serve as a soft boundary $\left(\partial E_{z} / \partial n=0\right)$; the boundary conditions switch roles for transverse magnetic (TM) waves.

The scattering properties of infinitely long cylinders are somewhat peculiar due to the long-range nature of the Green's function in two-dimensional space. For instance, the total scattering cross section (SCS) of a circular PEC cylinder in TE polarization (hard boundary) diverges as [27]

$$
\sigma_{\mathrm{sc}} \approx \frac{\lambda \ln \left(\frac{a}{\lambda} \pi \mathrm{e}^{\gamma}\right)}{\ln ^{2}\left(\frac{a}{\lambda} \pi \mathrm{e}^{\gamma}\right)+\left(\frac{\pi}{2}\right)^{2}} \sim \frac{\lambda}{\ln (a / \lambda)}
$$

in the long-wavelength limit $(\lambda \rightarrow \infty)$; here, $a$ is the cylinder radius and $\gamma$ is the Euler constant. This divergence is evident from the finite-element calculation of the SCS of a cloaked cylinder, shown in figure 1(a). In the simulation, the meshed cloak 'shrinks' its interior object to a finite size, proportional to the size of mesh elements near its interior boundary; therefore, the SCS diverges in the electrostatic limit no matter how small the mesh elements are. On the other hand, with a fixed $\lambda$ the SCS (4) converges to zero in the limit $a \rightarrow 0$. Therefore, a perfect cloak would still eliminate the SCS of a hard scatterer, since it compresses the electromagnetic width of the cylinder to zero.

However, the convergence of the SCS versus $a$ is so slow (logarithmic) that, in practice, or even in numerical simulations, perfect cloaking may never be accomplished with a hard boundary due to either the granularity of metamaterials or the finite size of the simulation mesh. The poor convergence of the SCS of a cloaked hard scatterer with respect to mesh size is illustrated by figure 1(a). In contrast to the cloaked hard scatterer, a 2D cloak with a soft boundary condition has a much smaller SCS to begin with and a much faster convergence with the mesh size (figure 1(b)). This issue is particular to two dimensions; three-dimensional spherical cloaks (figure 2) are not sensitive to the choice of interior boundary condition.

Unless specified otherwise, we will use the soft boundary condition in this section to decouple this issue from all other effects. Throughout this section, the SCS is normalized relative to the geometric cross section of the cloaked region, $\sigma_{\text {geom }}=$ $2 a$.

\subsection{Role of continuum discretization}

Mathematically, the continuous distributions of equation (1) substituted into the Helmholtz equation lead to zero scattered fields outside the cloak. All suggested practical implementations, however, involve approximating these continuous distributions with finite-size metamaterial cells. For cloaks obtained by radial transformations, such cells are typically arranged into concentric layers to retain the cylindrical symmetry of the device. Realistic cloak designs will inevitably include further discretization of each layer into unit cells; this would reduce the continuous rotational 

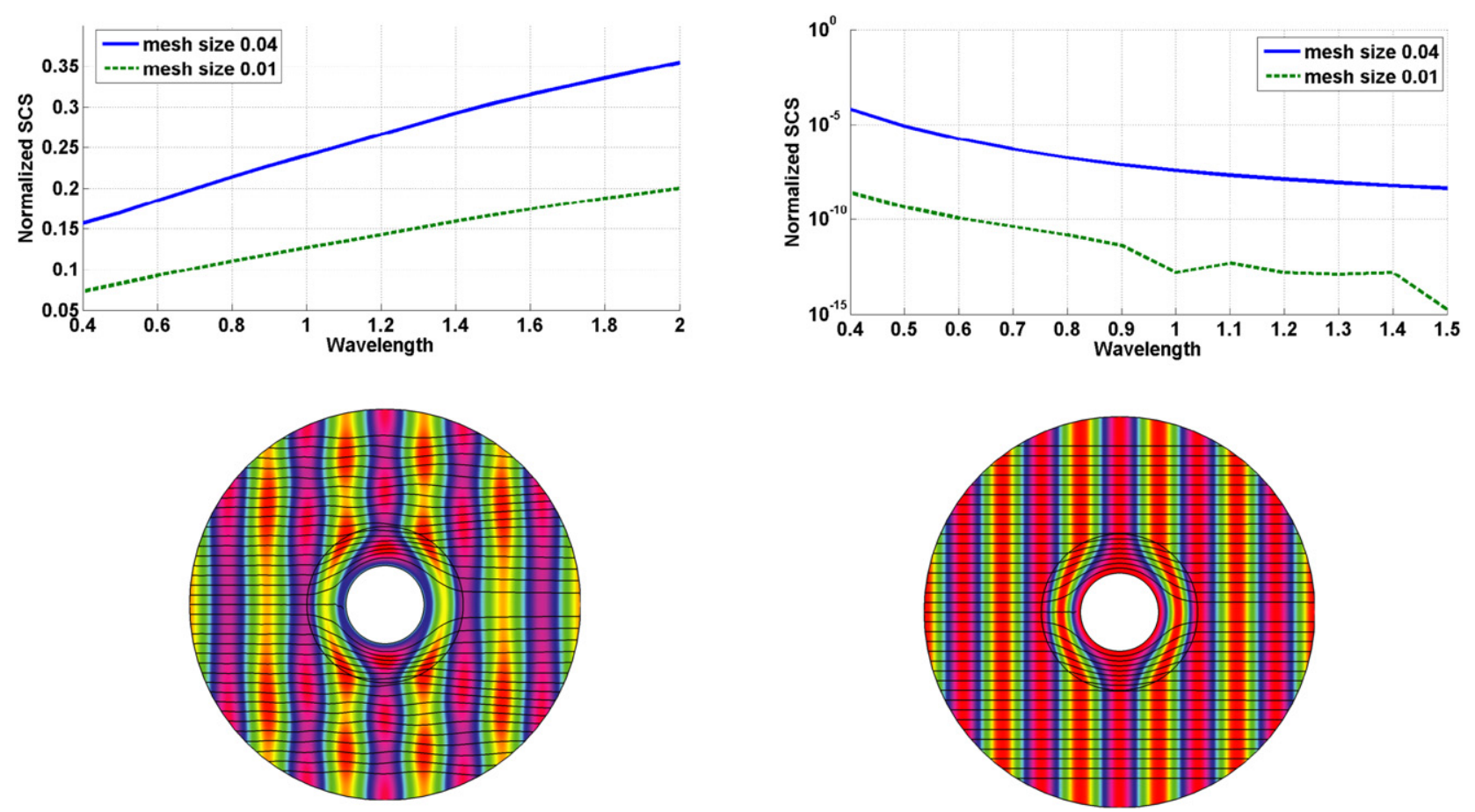

(a)

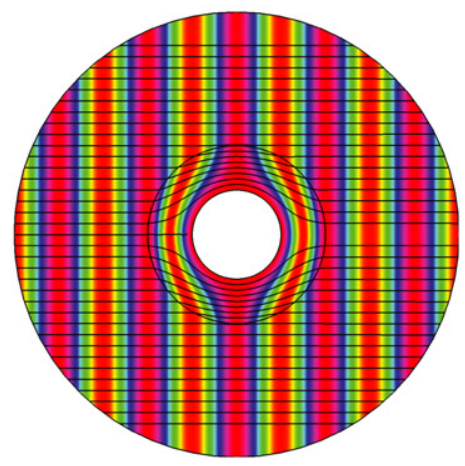

(b)

Figure 1. Two-dimensional perfect cloak defined by equation (1) with the linear transformation (3). The graphs show the total scattering cross section assuming (a) hard and (b) soft boundary conditions on the inner surface of the cloak, as a function of the wavelength. The images underneath the graphs show the electric field distributions at the wavelength $\lambda=0.4$. The cloak inner and outer radii are $a=0.2$ and $b=0.4$, respectively. The normalized SCS is defined as the ratio $\sigma_{\mathrm{sc}} / \sigma_{\mathrm{geom}}$, where $\sigma_{\mathrm{geom}}=2 a$ is the geometric width of the cloaked region. Numerical simulation performed with COMSOL Multiphysics.

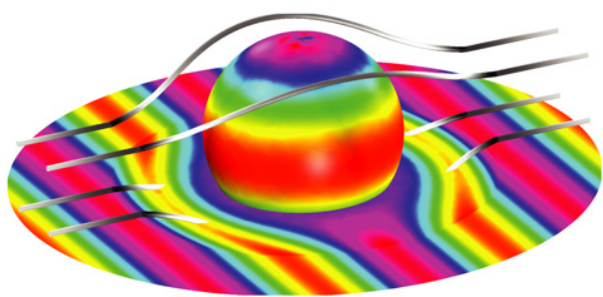

(a)

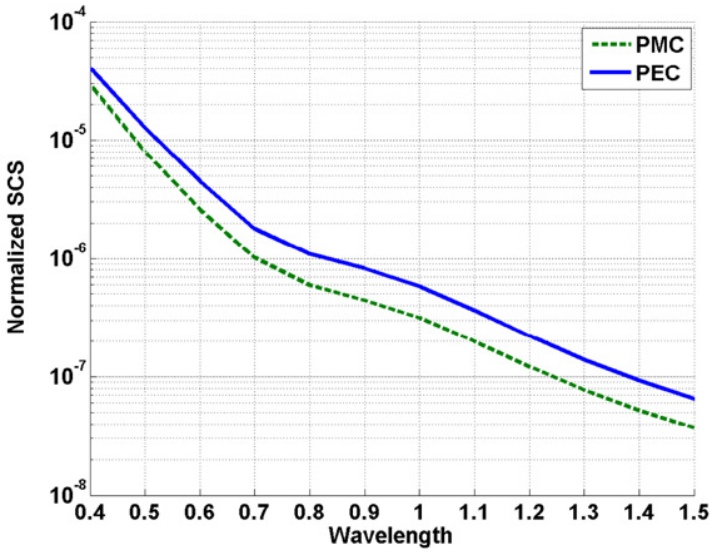

(b)

Figure 2. Three-dimensional perfect cloak defined by equation (2) with the linear transformation (3). Plots show (a) field profile and streamlines of electromagnetic flux at the wavelength $\lambda=0.4$ and (b) the total SCS with hard (PEC) and soft (PMC) boundary conditions, as a function of the wavelength. The cloak inner and outer radii are $a=0.2$ and $b=0.4$.

symmetry of the cloak (point group $D_{\infty}$ ) to discrete rotational symmetry (group $D_{n}$ ), or even lesser symmetry. Here, we study only the effect of radial discretization, assuming that the effective medium parameters are stepwise functions of the radius and independent of the azimuthal angle. We show that, although all choices of the transformation function $q(r)$ may be equivalent in the continuum limit, the performance of a cloak based on those choices will depend on the particular discretization applied. Figure 3 demonstrates monotonic convergence of the total SCS of a layered cloak to zero in the limit of the large number of layers $(N \rightarrow \infty)$; this behavior is expected and it exists at any wavelength-to-diameter ratio of the cloak.

Figure 4 shows the results of numerical simulations of a layered cloak that uses a family of transformation functions:

$$
q(r)=b(r-a)(1+p(r-b) / b) /(b-a),
$$




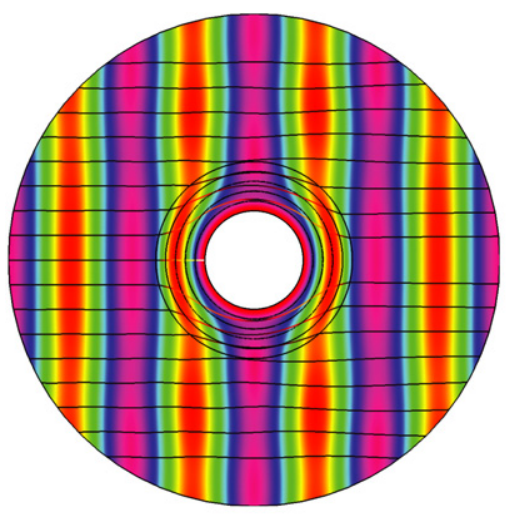

(a)

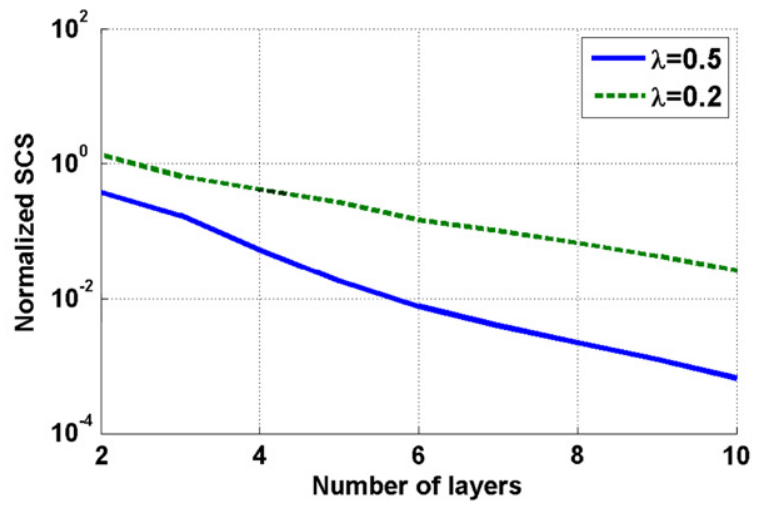

(b)

Figure 3. The layered version of the perfect 2D cloak shown in figure 1(b). The continuum transformation is discretized by evaluating the material parameters at the center of each layer and applying them uniformly to that entire layer. All layers have identical thickness. Plots show (a) field profile and streamlines of electromagnetic flux at the wavelength $\lambda=0.5$ for the number of layers $N=5$ and (b) the total SCS with a soft boundary condition on the interior, as a function of $N$, for two different wavelengths, $\lambda=0.5$ and 0.2 . The cloak inner and outer radii are, again, $a=0.2$ and $b=0.4$.

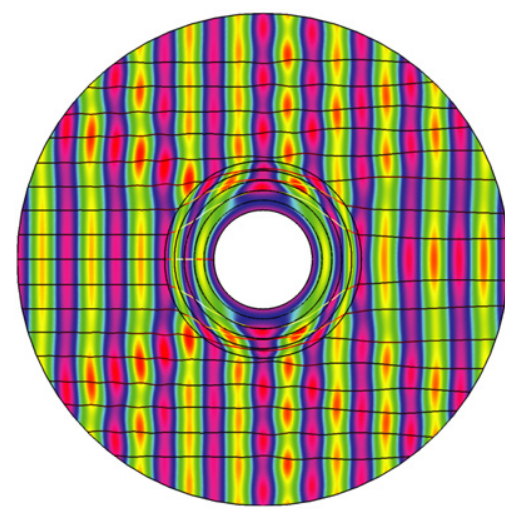

(a)

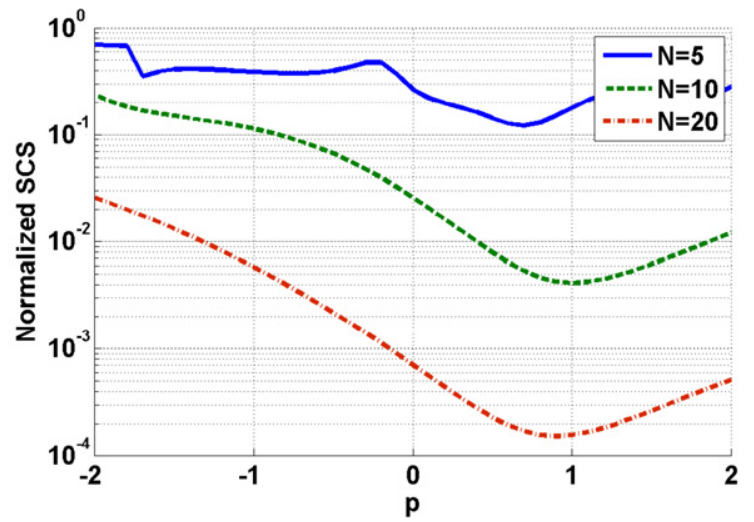

(b)

Figure 4. The layered version of the perfect $2 \mathrm{D}$ cloak corresponding to the family of transformations (5), as a function of the parameter $p$, at a fixed wavelength $\lambda=0.2$. Plots show (a) field profile and streamlines of electromagnetic flux at the parameter value $p=0.7$ and number of layers $N=5$, and (b) the total SCS as a function of the parameter $p$, for $N=5,10$ and 20. The SCS converges to zero in the limit $N \rightarrow \infty$ regardless of the value of $p$. However, for any finite number of layers it can be minimized by choosing a transformation function different from linear. The optimized value of $p$ depends on $N$, as well as the wavelength $\lambda$.

with fixed cloak dimensions $a$ and $b$, and one adjustable parameter $p$. At $p=0$, the usual linear radius transformation is obtained. Our parametric analysis shows that the total SCS of a layered cloak can be improved by optimizing the value of $p$. The optimized value of $p$ depends on $N$, as well as the wavelength $\lambda$. The optimum value of $p$ is typically quite different from zero, meaning that the linear transformation is not the optimum. For example, in a cloak with $N=5$ layers, the SCS at $\lambda=a=0.2$ can be reduced by a factor of two, compared to its value at $p=0$, by choosing $p=0.7$. Similar improvement is observed for layered cloaks with larger $N$. Worth mentioning is the convergence of the SCS towards zero with increasing values of $N$, which happens at any value of $p$. This behavior confirms that all transformations in the family (5) are equivalent in the continuum limit, though they are not quite the same for any finite $N$. Further improvement of the SCS can be obtained by general optimization of material properties in each layer, without restriction to any particular class of transformations [28].

\subsection{Exploring transformation freedom}

The freedom of choice of the transformation function $q(r)$ can be exploited to make one or more material properties constant throughout the cloak. For example, a transformation that leads to a perfect TM-wave cloak with $\mu_{z}=$ const is

$$
\begin{gathered}
q(r)=b \sqrt{r^{2}-a^{2}} / \sqrt{b^{2}-a^{2}}, \\
\mu_{z}=b^{2} /\left(b^{2}-a^{2}\right), \quad \epsilon_{r}=1 / \epsilon_{\theta}=\left(r^{2}-a^{2}\right) / r^{2} .
\end{gathered}
$$

Even more curious is the fact that two parameters can be kept constant simultaneously: the family of transformations 
from [23]

$$
\begin{gathered}
q(r)=b\left(\frac{r}{b}\right)^{C}, \quad \mu_{\theta}=1 / \mu_{r}=C, \\
\epsilon_{z}=C\left(\frac{r}{b}\right)^{C-1},
\end{gathered}
$$

where $C>1$ is an arbitrary constant, implements TEwave cloaks with constant magnetic permeability tensors. We observe, however, that a cloak given by equations (7) is imperfect for any finite value of shape aspect ratio $b / a$ : it compresses the apparent size of the cloaked region to a cylinder of finite radius $q_{\min }=b\left(\frac{a}{b}\right)^{C}>0$. Fortunately, this radius decreases exponentially as a function of $C$; thus (7) is a good approximation to a perfect cloak, as long as $C \gg 1$ and $a / b<$ 1 is not too close to unity. In practice, due to divergence of material properties of a perfect cloak at $r=a$, a cutoff radius is always introduced; in this sense, there is little difference between the 'perfect' cloak given by (6) and the 'imperfect' cloak (7).

In both cases (6) and (7) there are inequalities constraining the values of the constants $\mu_{z}>1$ and $\mu_{\theta}>1$; most unfortunately, they cannot be unity. The latter choice is, however, possible if an additional approximation is introduced, as described in section 2.4.

\subsection{Eikonal approximation and the role of boundary conditions}

Controlling all six of the constitutive tensor elements for a three-dimensional cloak, or three of the elements for a twodimensional cloak, may be difficult to achieve in practice. Controlling magnetic parameters is particularly challenging, especially in the optical frequency range [22, 19]. An approximation to the perfect cloak based on the geometrical optics (GO) limit was introduced to facilitate the experimental demonstration [14]. Monochromatic light propagation in the GO limit is well described by the eikonal equation [29] and ray tracing [30]. In the GO (short-wavelength) limit, the streamlines of electromagnetic flux are approximately coincident with the ray trajectories obtained via ray-tracing techniques, and the surfaces of constant phase of nonvanishing field components are approximated by the constantphase surfaces calculated with the optical eikonal equation. Neglecting the difference between those approximately equal quantities in the limit $\lambda \rightarrow 0$ is known as the eikonal approximation. We refer to the cloaks that rely on the eikonal approximation as eikonal cloaks. The eikonal approximation to the perfect cloak is obtained through the following procedure.

First, note that the refractive indices corresponding to radial $\left(n_{r}\right)$ and azimuthal $\left(n_{\theta}\right)$ propagation in the perfect cloak (1) are

$$
n_{r}=q^{\prime}, \quad n_{\theta}=q / r,
$$

for both TE and TM polarizations in 2D. For TM-polarized in-plane waves, the refractive indices are $n_{r, \theta}^{\mathrm{TM}}=\sqrt{\epsilon_{\theta, r} \mu_{z}}$, whereas for TE waves $n_{r, \theta}^{\mathrm{TE}}=\sqrt{\mu_{\theta, r} \epsilon_{z}}$.

Interestingly, the relationships (8) also hold for 3D cloaks with parameters (2). This is so because, in the limit $\lambda \rightarrow 0$, planar rays do not feel the gradient of refractive index transverse to the plane of propagation; any cross section of a 3D cloak passing through its center is equivalent to a cylindrical (2D) cloak in the GO limit.

By definition, an eikonal cloak uses the less restrictive set of requirements (8) instead of the full set (1) or (2). When light propagation is governed by the eikonal equation, wave impedance is relatively unimportant (to the extent described below) and the refractive index defines the ray trajectories, as well as the streamlines of electromagnetic flux.

The eikonal equation is, however, unable to describe the dynamics of light at an interface where wave impedance has a discontinuity. Reflection and transmission on such a discontinuity is governed mostly by impedance, which does not play a role in the eikonal equation. The perfect cloak may introduce a discontinuity in $\epsilon$ and $\mu$ at $r=b$ (if $q^{\prime}(b) \neq 1$ ): however, the relevant wave impedance $Z_{\theta}$, defined as $\sqrt{\mu_{z} / \epsilon_{\theta}}$ or $\sqrt{\mu_{\theta} / \epsilon_{z}}$ depending on the polarization, is always continuous in a perfect cloak: $Z_{\theta}=q / r$ matches unity at $r=b$.

There are infinitely many ways to reduce a perfect cloak that uses a given transformation function $q(r)$ to an eikonal cloak with the same $q(r)$. Depending on how the eikonal approximation is introduced, and what $q(r)$ is used, $Z_{\theta}$ may or may not be continuous at $r=b$. The most common way of reducing a perfect cloak to an eikonal cloak is to assume that one of the three material parameters is prescribed by an arbitrary function of radius; then the other two material properties are expressed through that function using equations (8). The most natural choice of such an arbitrary function is a constant; for instance, unity. The TE-wave cloak from $[14,26,15]$ (case A) assumes $\mu_{\theta}(r)=1$ for $a<r<b$, and consequently

$$
\begin{gathered}
\epsilon_{z}=n_{r}^{2} / \mu_{\theta} \rightarrow n_{r}^{2}=\left(q^{\prime}\right)^{2}, \\
\mu_{r}=n_{\theta}^{2} / \epsilon_{z}=\mu_{\theta} n_{\theta}^{2} / n_{r}^{2} \rightarrow n_{\theta}^{2} / n_{r}^{2}=(q / r)^{2} /\left(q^{\prime}\right)^{2} .
\end{gathered}
$$

The TM cloak of $[22,19]$ (case B) uses $\mu_{z}(r)=1$ for $a<r<b$; other parameters become

$$
\begin{gathered}
\epsilon_{r}=n_{\theta}^{2} / \mu_{z} \rightarrow n_{\theta}^{2}=(q / r)^{2}, \\
\epsilon_{\theta}=n_{r}^{2} / \mu_{z} \rightarrow n_{r}^{2}=\left(q^{\prime}\right)^{2} .
\end{gathered}
$$

We emphasize that eikonal cases A and B are fundamentally different from the perfect cloak given by (6).

Cases A and B, though distinct, are quite similar in terms of their overall performance. With a linear transformation function (3), case A has impedance mismatch on the surface:

$$
Z_{\theta}(b)=\sqrt{\mu_{\theta} / \epsilon_{z}}=1 / q^{\prime}(b)=(b-a) / b ;
$$

case B has the same mismatch:

$$
Z_{\theta}(b)=\sqrt{\mu_{z} / \epsilon_{\theta}}=1 / q^{\prime}(b)=(b-a) / b .
$$

Case A has been studied extensively in $[14,15,26]$; our calculations for case B are shown in figure 5.

The simulations in figure 5 confirm our suspicion that eikonal cloaks with impedance mismatch at the surface do not operate very well, even in the limit $\lambda \ll a$ where the 


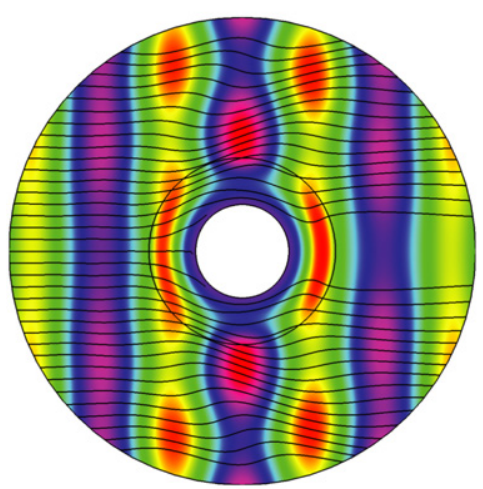

(a)

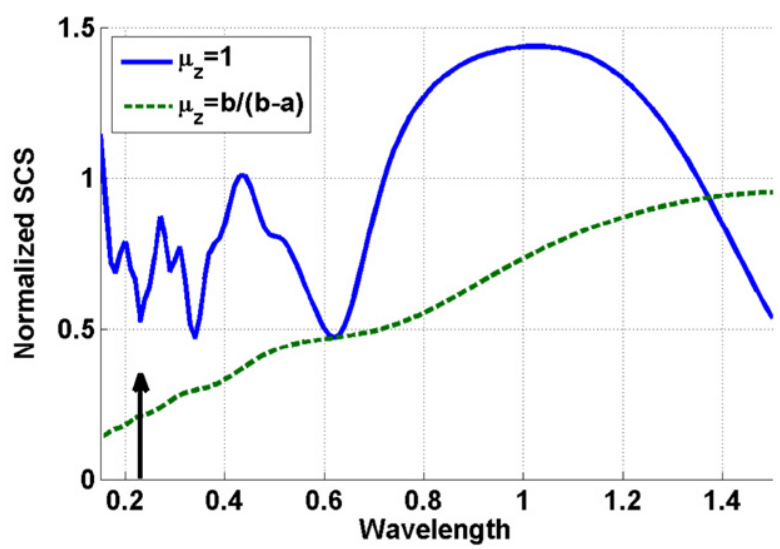

(b)

Figure 5. Two-dimensional eikonal cloak for TM polarization corresponding to the linear transformation of radius. The eikonal approximation is obtained by setting the out-of-plane magnetic permeability $\mu_{z}$ to one. A soft (Neumann) interior boundary condition is used $\left(\partial H_{z} / \partial n=0\right)$; this corresponds to a PEC boundary. Wave impedance at the exterior boundary is $Z_{\theta}(b)=\mu_{z}(b-a) / b=(b-a) / b<1$. Plots show (a) field profile and streamlines of electromagnetic flux at the wavelength $\lambda=0.6$ where SCS has a minimum and (b) the total SCS as a function of the wavelength. For comparison, graph (b) also shows SCS of an eikonal cloak with surface impedance matched to free space, $Z_{\theta}(b)=1$; this is done by choosing $\mu_{z}=b /(b-a)$. The arrow at $\lambda=0.22$ indicates the regime $(\lambda / a=1.107, b / a=2)$ in which eikonal cloaking was demonstrated in $[30,15]$. Direct comparison of SCS value with [15] cannot be made as it used the hard boundary (PEC for TE waves) with a much larger uncloaked SCS. The total SCS of an ideal cloak (not shown here, see figure 1 (b)) is less than $10^{-3}$ for all wavelengths depicted.

eikonal approximation is expected to be valid. One way to alleviate this problem is to choose the value of $\mu_{z}=$ $b /(b-a)$ such that $Z_{\theta}(b)=1$; the transformation function need not change. The eikonal cloak with corrected impedance performs very well; this was shown in [19] where a different approach (making the transformation function $q(r)$ continuous along with its first derivative) was suggested for impedance matching. The surface-impedance-matched eikonal cloak shows a linear decrease of the total SCS in the limit $\lambda \rightarrow 0$, as seen from figure $5(\mathrm{~b})$.

This finding may seem to contradict the earlier claim [26] that eikonal cloaks are not invisible in the GO limit. The apparent contradiction can be better understood by considering the multipole contributions to the SCS, which can be readily found for cylindrically or spherically symmetric structures. It was found in [26] that, for a cylindrical cloak, at least one scattering coefficient in the Bessel expansion formalism remains non-zero and finite in the limit $b / \lambda \rightarrow \infty$. This poses no contradiction to the findings of this work and of [22]. The total SCS expression through the scattering coefficients [27] has an overall factor of the order of $\lambda$ :

$$
\sigma_{\mathrm{sc}}=\frac{2 \lambda}{\pi} \sum_{m=-\infty}^{\infty}\left|b_{m}\right|^{2} .
$$

Here, $b_{m}$ are the complex-valued scattering coefficients for TE polarization.

For the eikonal cloak with corrected surface impedance, the sum in equation (13) remains finite (does not diverge) in the limit $\lambda \rightarrow 0$; the SCS thus converges to zero as $\mathrm{O}(\lambda)$, as is evident from figures 5(b), 6(b) and 7(b). In simple terms, such a cloak is almost invisible to an observer who can only measure the far fields. In contrast, when the cloaking shell is removed and the scattering object (a perfectly reflecting cylinder) is exposed to a plane wave in free space, the sum in equation (13) diverges as $1 / \lambda$ at $\lambda \rightarrow 0$, leading to a finite scattering width in the GO limit [27]. One may therefore state that, although eikonal cloaks are much worse than perfect cloaks (the latter have $b_{m}=0$ for any $\lambda$ and $m$ ), their SCS is anomalously small in comparison with a regular scatterer of the same size.

So far we have studied cloaks with an insulation condition, either a soft (Neumann) or hard (Dirichlet), at the interior boundary. For some applications it may be desirable to allow fields to leak into the cloak interior. This is, in fact, the case assumed in [26]. Mathematical analysis of the exact Helmholtz equation for an eikonal cloak from case A has been performed therein. It was shown that the monopolar $(m=0)$ Bessel harmonic leaks into the cloaked region of an eikonal cloak, whereas any harmonics with $m>0$ do not penetrate at all. This is clearly illustrated by figure 6(a), which shows azimuthally symmetric fields inside the cloak. The SCS calculation (figure 6(b), solid curve) confirms the finding of [26] that $m=0$ harmonics contribute to the SCS, causing the oscillations of $\sigma_{\mathrm{sc}}$ as a function of $\lambda / b$. Nevertheless, even the air-filled, non-insulated eikonal cloak still shows convergence of SCS to zero. The convergence is, however, not uniform in $\lambda / b$, contrary to the case of an eikonal cloak with soft insulating boundary (figure 6(b), dashed curve).

Finally, following [19], we note that surface impedance matching of an eikonal cloak-a prerequisite for its invisibility—can be achieved without controlling $\epsilon_{z}$ or $\mu_{z}$. For visible wavelengths, making magnetic materials with $\mu_{z} \neq 1$ and a low loss tangent is difficult. To avoid this challenge, one may choose a more appropriate transformation function $r^{\prime}=q(r)$. According to equation (12), the wave impedance for transverse waves incident on the eikonal cloak with $\mu_{z}=1$ and $\epsilon_{\theta}=\left(q^{\prime}\right)^{2} / \mu_{z}=\left(q^{\prime}\right)^{2}$ reduces to $Z_{\theta}=1 / q^{\prime}$. Thus, any transformation with $q^{\prime}(b)=1$ accomplishes surface impedance matching in eikonal cloaks. 


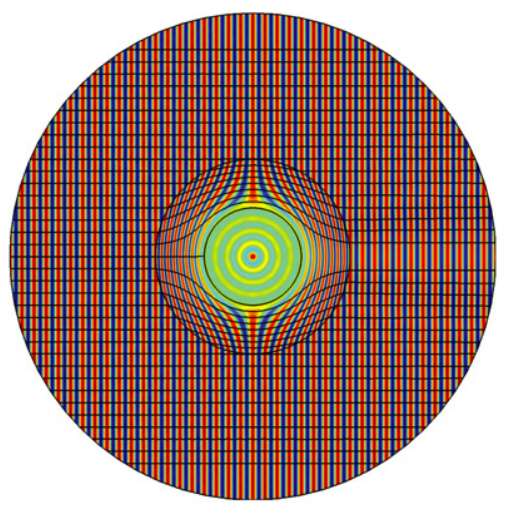

(a)

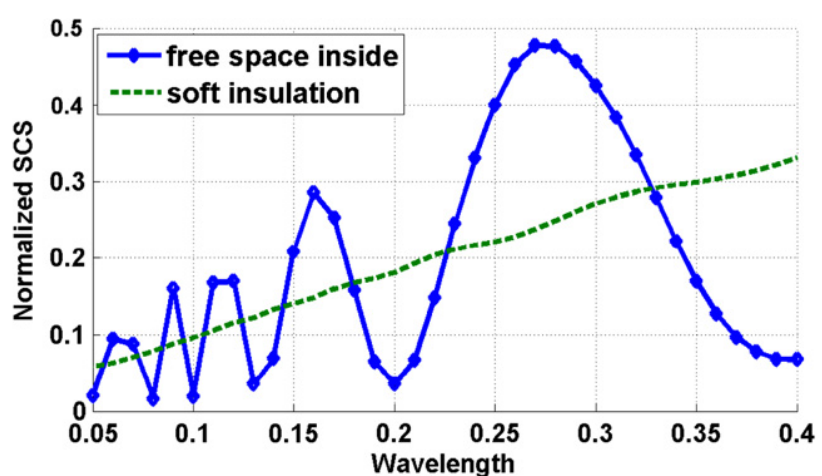

(b)

Figure 6. The same eikonal cloak for TM polarization as in figure 5, but without an insulating interior boundary. The interior of the cloak is free space $(\epsilon=\mu=1)$ and the interior boundary at $r=a$ is an electromagnetic continuity condition. The wave impedance at $r=b$ is matched to free space by choosing $\mu_{z}=b /(b-a)$ in the annulus $a<r<b$. Plots show (a) field profile and streamlines of electromagnetic flux at the wavelength $\lambda=0.05$ and (b) the SCS as a function of the wavelength. The field plot indicates that the $m=0$ angular harmonics penetrate into the core of the cloak, while harmonics with $m>0$ do not [26]. Due to transmission of $m=0$ harmonic, the SCS of this structure (solid blue curve) shows oscillatory behavior in the regime $\lambda<a$, in stark contrast with the linear decrease of $\sigma \propto \lambda$ for a cloak with a soft insulating boundary at $r=a$ (dashed green curve). The absolute values of scattering efficiency in the regime $\lambda \ll a$ are comparable for both cloaks.

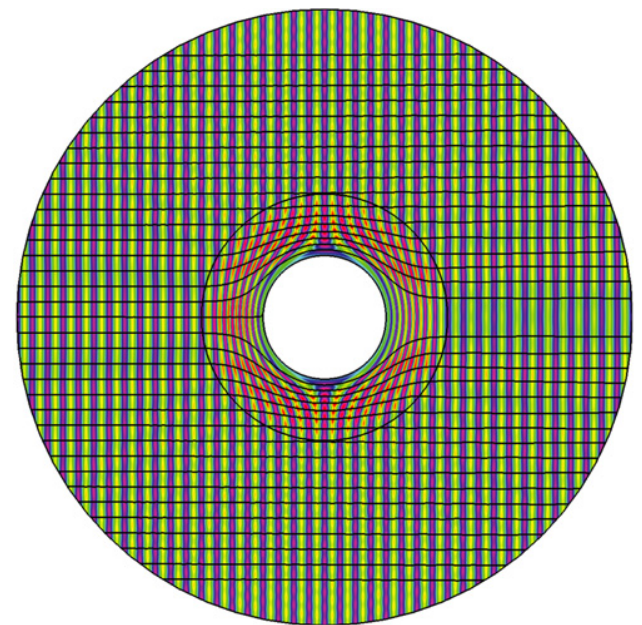

(a)

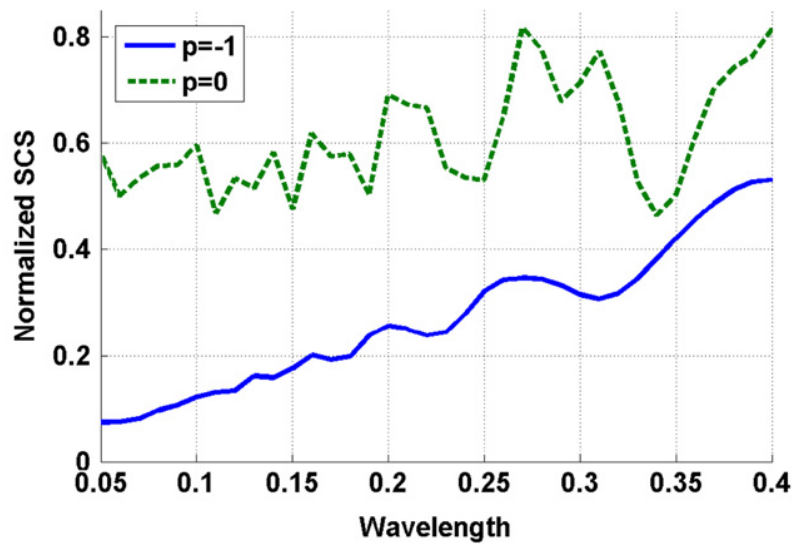

(b)

Figure 7. A TM-wave eikonal cloak that uses a quadratic transformation of radius (5) with $p=p_{\text {opt }} \equiv-a /(b-a)=-1$. All other parameters are the same as in figure 5; out-of-plane permeability $\mu_{z}=1$. Plots show (a) field profile and streamlines of electromagnetic flux at the wavelength $\lambda=0.05$ and (b) the total SCS as a function of the wavelength. The total SCS of an un-optimized eikonal cloak (with $p=0$ ) (same as in figure 5) is also shown for comparison. The SCS of an ideal cloak (not shown) is less than $10^{-3}$ in this entire band, regardless of the value of $p$.

One family of cloaking transformations that can be optimized to get $Z_{\theta}(b)=1$ is the quadratic polynomial (5). For $p=0$, it reduces to the linear transformation (3). The requirement $Z_{\theta}(b)=1$ is fulfilled at $p=-a /(b-a)$. A finite-element simulation (figure 7) confirms that the SCS of an eikonal cloak with $\mu_{z}=1$ is strongly reduced at this value of $p$, versus its value at $p=0$. We also note that $\sigma_{\mathrm{sc}}$ decreases approximately linearly with $\lambda$ in the range $0.05 \leqslant \lambda \leqslant 0.4$. This behavior is similar to what is observed in figure $5(\mathrm{~b})$ for a cloak whose impedance was matched by adjusting $\mu_{z}$. The two examples in figures 5(b), 7(a) and (b) clearly show that eliminating impedance mismatch at the exterior boundary of an eikonal cloak is a necessary condition for its low visibility.

\section{Optical conformal mapping approach}

Optical conformal mapping (CM) is closely related to the TO approach in that they both utilize geometry for the purposes of electromagnetic design [16, 31]. CM techniques are based on the premise that, under conformal coordinate transformations, the two-dimensional Helmholtz equation is form-invariant, just as Maxwell's equations are form- 


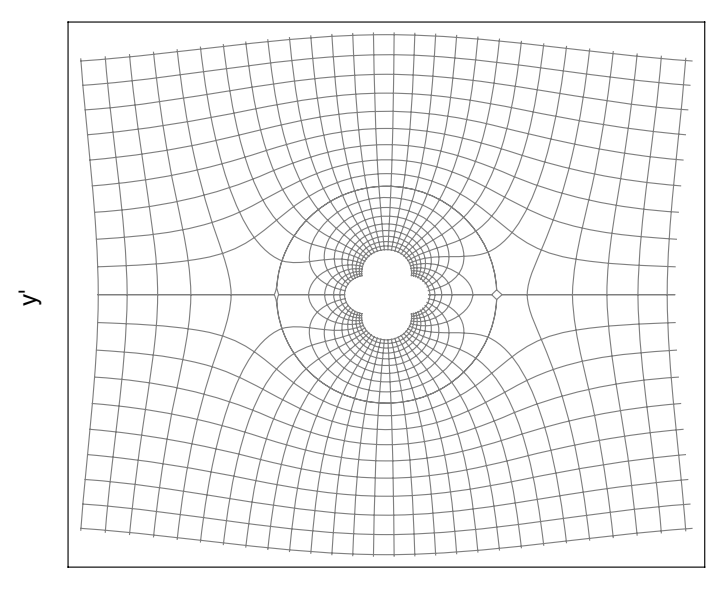

$x^{\prime}$

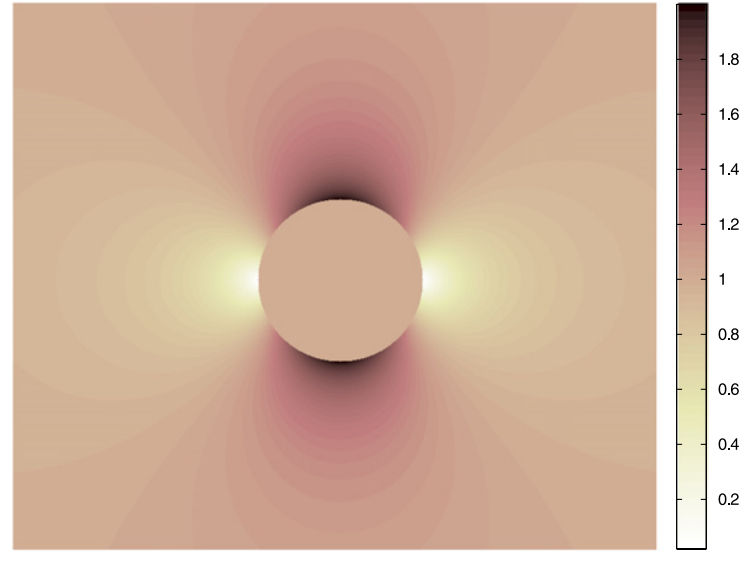

(b)

Figure 8. The (a) coordinate transform and (b) index profile are shown for the cloak resulting from an optical conformal map.

invariant under arbitrary transformations. Analogous to TO, where the coordinate transformation is used to modify the permittivity and permeability tensor distributions, in CM the coordinate transformation is used to redefine the refractive index distribution. Unlike the most general TO media, CM media can be dielectric only and isotropic, and thus have the potential to scale more easily towards visible wavelengths. The simplicity of material properties in CM, however, is accompanied by significantly reduced performance, as might be expected from the restricted material parameter set.

In the context of optical conformal mapping, the Helmholtz equation was originally utilized for managing rays in the GO (eikonal) limit [16, 31]. For any wave propagating in a two-dimensional plane, the polarization is maintained as TE (TM), if both $\epsilon$ and $\mu$ tensors are diagonal. If one further assumes that $\mu=1$ (respectively, $\epsilon=1$ ), only the non-trivial out-of-plane permittivity $\epsilon_{z z}$ (or permeability $\mu_{z z}$ ) remains. Maxwell's equations then reduce to a scalar Helmholtz equation $\nabla^{2} \Psi+(\omega / c)^{2} n^{2} \Psi=0$, where $\Psi=$ $E_{z}\left(H_{z}\right)$. This equation is invariant under any conformal transformation. Thus, for a single polarization case, the CM approach is exact from the full-wave perspective, and not just in the eikonal approximation.

The main advantage of the CM technique is that, in order to control the in-plane propagation of TE (TM) waves, it is sufficient to provide only the isotropic permittivity (permeability) distribution. In two dimensions, the theory of functions of complex variables suggests a rich class of conformal transformations. The in-plane axes in the original and transformed spaces can be treated as the real and imaginary parts of complex-valued coordinates, $z$ and $z^{\prime}$, respectively; then, any holomorphic complex function $z^{\prime}=f(z)$ generates a conformal map.

Unfortunately, finding a conformal transformation that establishes a mapping between two given finite-area domains is often impossible [32]. One reason for this is that conformal maps preserve the so-called conformal modulus of the domain, which is a dimensionless characteristic of its shape; therefore, any two areas of unequal conformal modulus cannot be mapped conformally [33]. A variety of numerical techniques have emerged for obtaining approximately conformal solutions, called quasi-conformal [32, 34], which minimize the amount of anisotropy in the constitutive parameters subject to the particular boundary conditions. On the other hand, mapping conformally the exterior of a circle onto the exterior of another circle with a different radius is not prohibited, since both domains involved have an infinite conformal modulus. The distribution of refractive index that implements such a mapping precisely would be, of course, infinite in extent.

The optical CM technique was originally introduced with the goal of constructing a medium via a conformal transformation for which rays would be guided around an internal cylindrical object [16]. With conformal transformations, it is not possible to find an azimuthally symmetric solution to this problem, nor is it possible to achieve any configuration of isotropic media that would scatter precisely as a volume of free space [16-18]. For rays incident from a particular direction, however, a conformal map can be found that suggests, in the geometrical optics limit, a very similar ray behavior as was observed for the TO cloaks. One such conformal transformation is given by [16]

$$
w=z+a^{2} / z
$$

where $a$ is the radius of the inner cylinder, and $w=x+\mathrm{i} y$ and $z=x^{\prime}+\mathrm{i} y^{\prime}$ are the complex coordinates in the two frames. Note that, since $w(z)$ is a holomorphic function (i.e. $\left.\partial w / \partial z^{*}=0\right)$, the transformation is conformal. The map specified by equation (14) is illustrated in figure 8(a), where lines of constant $x$ and constant $y$ are plotted in the $\left(x^{\prime}, y^{\prime}\right)$ space. The plot shows that rays traveling in a straight line in the $(x, y)$ space follow curvilinear trajectories in the $\left(x^{\prime}, y^{\prime}\right)$ space, either detouring around the central cylinder if incident along the horizontal axis, or terminating on the cylinder if incident along the vertical axis. The effective index distribution that implements the mapping for the Helmholtz equation can be 


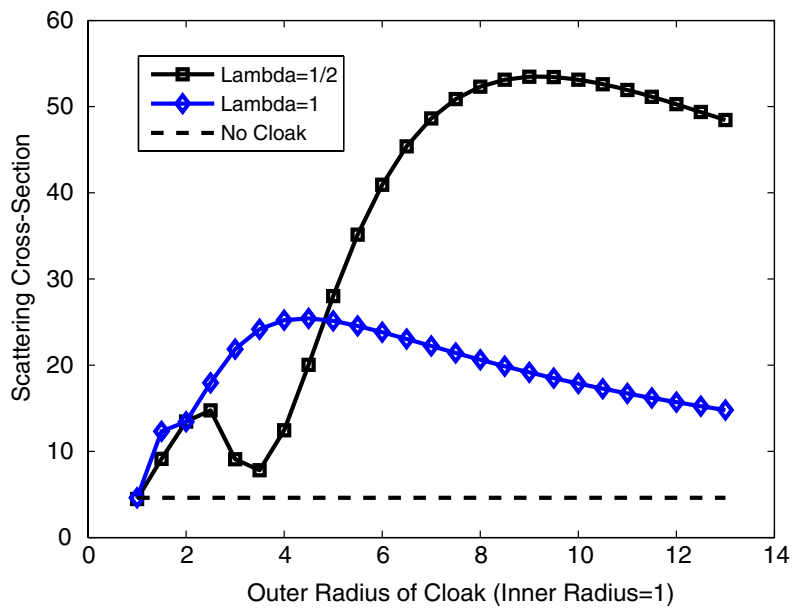

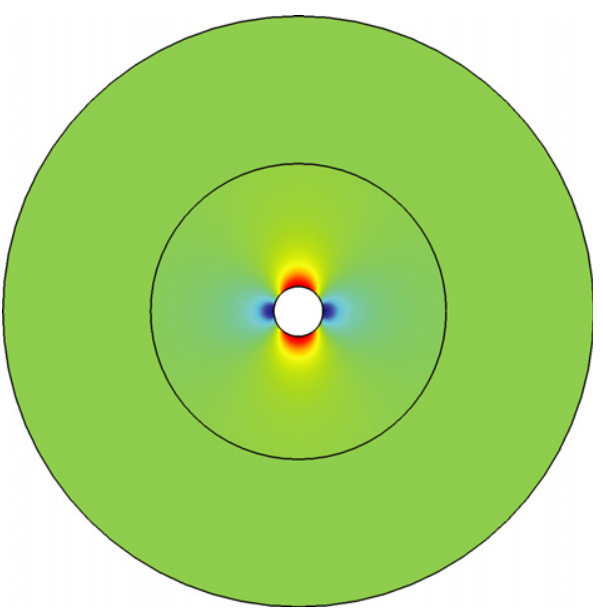

(b) (a)

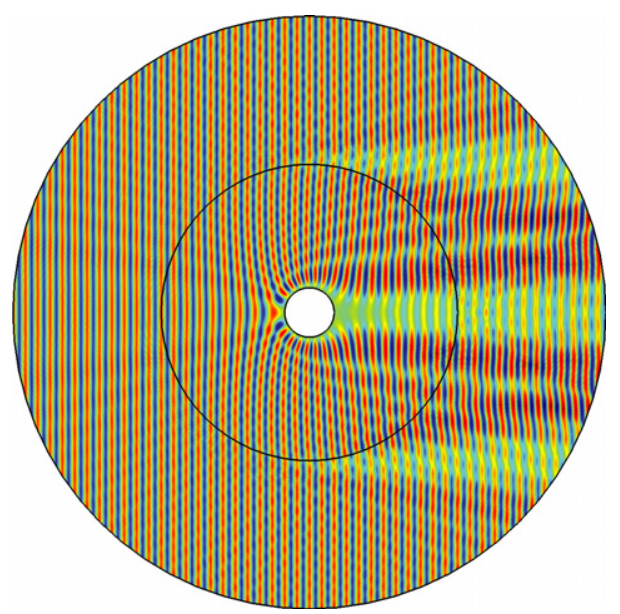

(c)

Figure 9. Full-wave simulations of the conformal cloak with various sizes. (a) The SCS (in arbitrary units) as a function of the cloak outer radius $b$ for two wavelengths, $\lambda=1$ and 0.5 , assuming $a=1$. (b) The index profile for a cloak with the shape aspect ratio $b / a=6$. The refractive index mismatch at the boundary $r=b$ is less than $10 \%$, yet it affects the cross section. (c) Full-wave simulation for the same cloak with $b / a=6$. Refraction at the outer boundary and shadowing around the core cylinder are evident; these effects contribute to the SCS.

directly calculated using $n=|\mathrm{d} w / \mathrm{d} z|$ :

$$
n(x, y)=\left|1-\frac{a^{2}}{z^{2}}\right| \text {. }
$$

This transformation can be equivalently written as

$$
\begin{gathered}
x^{\prime}(x, y)=x \pm \frac{1}{\sqrt{2}}\left(\sqrt{\left(x^{2}-y^{2}-4 a^{2}\right)^{2}+4 x^{2} y^{2}}\right. \\
\left.+\left(x^{2}-y^{2}-4 a^{2}\right)\right)^{1 / 2}, \\
y^{\prime}(x, y)=y \pm \frac{1}{\sqrt{2}}\left(\sqrt{\left(x^{2}-y^{2}-4 a^{2}\right)^{2}+4 x^{2} y^{2}}\right. \\
\left.-\left(x^{2}-y^{2}-4 a^{2}\right)\right)^{1 / 2} .
\end{gathered}
$$

The index distribution corresponding to equation (16), shown graphically in figure 8(b), successfully guides rays which are initially parallel to the $x$ axis around the inner cylinder. Both the transformation and the prescribed index distribution for the CM cloak, however, are infinite in spatial extent. The only free parameter in this transformation is the radius of the inner cylinder. In order to characterize the efficacy of such a cloak, even in simulation, the transformation must be approximated by applying a cutoff at some radius. Unfortunately, the cutoff radius introduces significant deleterious scattering, leading to a larger overall SCS. Fullwave simulations, performed in COMSOL, characterize the effects of approximating the infinite conformal cloak by a finite-sized version in which the index profile is terminated at a finite radius. The SCS is shown as a function of the terminated cloak size in figure 9 for two different wavelengths. These simulations do not demonstrate a reduction in SCS for a cloak of any size within our computational capabilities. Scattering is introduced at the boundary of the cloak, which produces a shadow, as demonstrated in figure 9. For this case the cloak increases the SCS of the inner cylinder by nearly a factor of ten despite less than $10 \%$ error in the refractive index at the boundary.

It should be expected that optical cloaks with larger outer radius exhibit better cloaking performance; for a very large radius, it is reasonable to employ Hamiltonian ray-tracing 


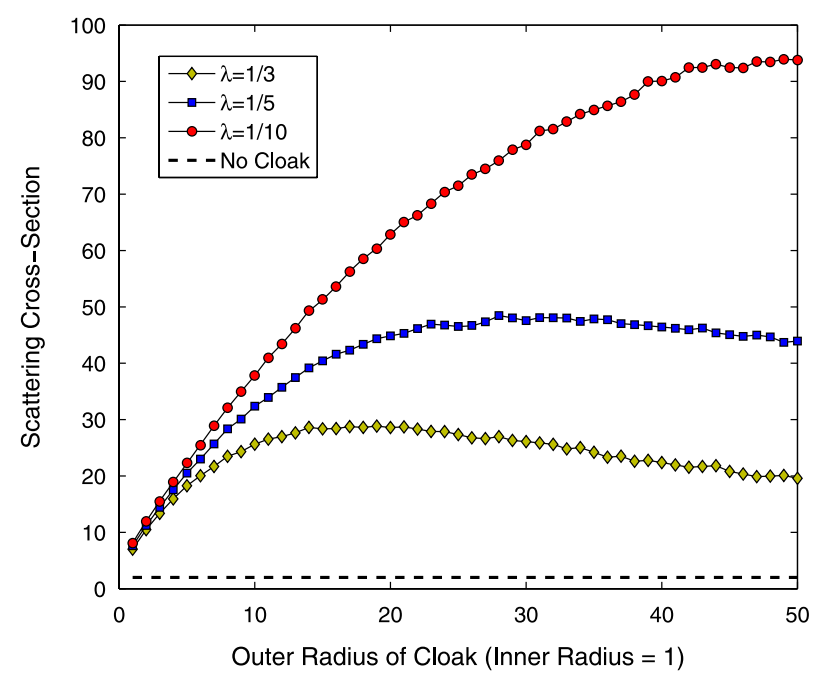

(a)

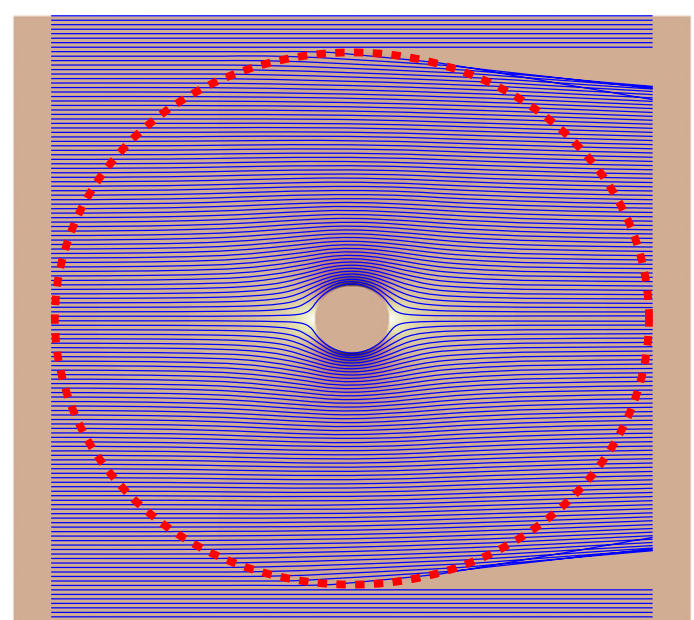

(b)

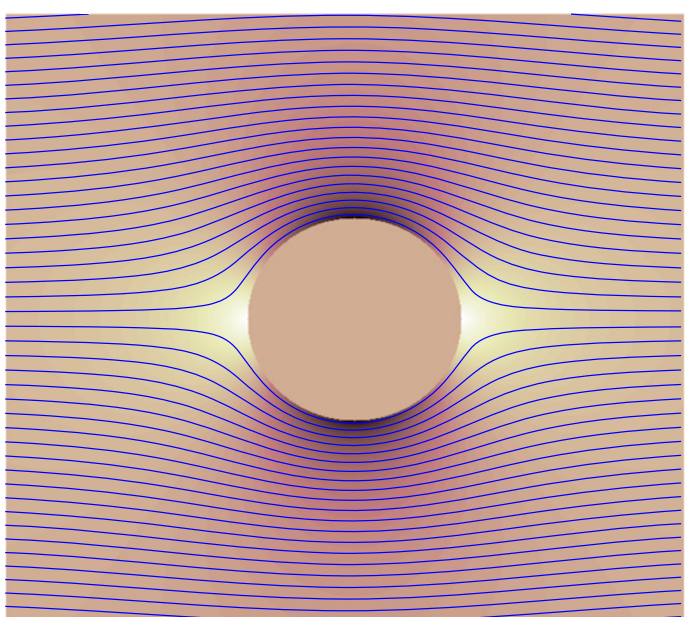

(c)

Figure 10. Ray-tracing simulation of an optically large conformal cloak. The SCS is calculated using the procedure described in the text. (a) The SCS as a function of outer radius $b$ for several wavelengths; the latter determine the cutoff scattering angle. Note that the SCS actually diverges in the limit $\lambda / b \rightarrow 0$. (b) and (c) Ray trajectories for an outer radius $b=6$. Rays near the origin are guided around the inner cylinder (c): however, there is noticeable refraction at the boundary of the cloak (b).

techniques [30]. The SCS in the GO limit may be defined as the total number of rays that are scattered from an object, divided by the density of rays. In our ray-tracing simulations, the density of incident rays is uniform. To distinguish between scattered and non-scattered rays, a minimum scattering angle, $\theta_{\min }$, must be introduced; without the angle cutoff, the integral (all-angle) SCS diverges for any potential that remains finite at all distances [35]. Rays deflected by an angle larger than this minimum are considered to be scattered rays and included in the total SCS calculation. Similarly, rays deflected by an angle less than $\theta_{\min }$ are considered remaining in the incident beam. A natural choice for the minimum scattering angle is the minimum resolvable half-angle, $\theta_{\min }=\frac{1}{2} \frac{\lambda}{d}$, from an aperture of diameter $d$. This choice explicitly relates the wavelength to the total SCS even when characterized in the GO approximation. For our calculation, we assume that the aperture size $d$ equals the diameter of the outer boundary of the cloak, $d=2 b$.
The SCS results from our ray-tracing analysis are shown in figure 10. The behavior of the SCS is in qualitative agreement with the full-wave simulations. Although rays close to the inner cylinder are appropriately guided around it, there is clear refraction on the outer boundary of the cloak. The SCS reaches a peak value and then begins to drop slowly as the size $b$ of the cloak is increased: however, no reduction in the SCS is found with any choice of wavelength. The conformal cloak with the finite ratio of outer to inner radius $b / a$ is therefore inherently visible at any wavelength.

We have confirmed via ray-tracing and full-wave simulations that this conclusion also applies to the device in which the transition from the conformal transformation region (the region filled with isotropic refractive index) to free space is graded continuously over a finite distance. The scattering off the truncation region of the cloak is therefore not removable by a simple graded-index approach that involves only isotropic dielectrics. 


\section{Conclusions}

TO and CM have ushered in a wave of creativity in electromagnetic design that has seen proposals for a host of novel devices. The 'invisibility cloak' has become the most widely recognized example for both techniques, and lends itself particularly well to quantitative analysis. Utilizing the total scattering cross section (SCS) as the measure of cloaking performance, we are able to evaluate and compare numerous TO and CM cloaking concepts. Using full-wave finite-element simulations, we have demonstrated that the so-called eikonal approximation cloaks feature anomalously small SCS. The SCS of eikonal cloaks is shown to vanish precisely in the GO limit if and only if the wave impedance is continuous at the exterior boundary. The role of the interior boundary is also clarified for two- and three-dimensional cloaks. It is explained why the hard insulation (Dirichlet) boundary condition leads to strong deviations from perfect cloaking in two dimensions. We have visualized penetration of the zeroth-order Bessel harmonic through an uninsulated interior boundary and shown the effect of transmission through the cloaked cavity. The convergence of TO cloaks with metamaterial granularity to perfect cloaks in the continuous material parameter limit is demonstrated. Several interesting choices of transformations leading to perfect and eikonal cloaks with one or more constant material properties are pointed out.

Using the total SCS as the figure-of-merit, we have also evaluated the performance of a non-rotationally invariant, isotropic-index cloak based on the optical conformal mapping method. It is shown using both full-wave simulations and Hamiltonian ray tracing that the choice of a cutoff radius strongly affects the SCS of conformal transformation cloaks, which remains finite for any size of cloak and any wavelength.

\section{Acknowledgments}

This work was partially supported through a Multiple University Research Initiative, sponsored by the US Army Research Office (contract no. W911NF-09-1-0539) and the US IC Postdoctoral Fellowship program. The authors are grateful to Magnus Olsson (COMSOL AB) for insightful conversations on computational electrodynamics.

\section{References}

[1] Pendry J, Schurig D and Smith D R 2006 Science 3121780

[2] Shalaev V M 2008 Science 322384

[3] Ward A J and Pendry J 1996 J. Mod. Opt. 43 773-793

[4] Nicolet A, Remacle J F, Meys B, Genon A and Legros W 1994 J. Appl. Phys. 756036
[5] Dolin L S 1961 Izv. Vyssh. Uchebn. Zaved. Radiofiz. 4964

[6] Post E G 1962 Formal Structure of Electromagnetics: General Covariance and Electromagnetics (New York: Interscience)

[7] Landau L D and Lifshitz E M 1975 The Classical Theory of Fields 4th edn (Amsterdam: Elsevier)

[8] Greenleaf A, Lassas M and Uhlmann G 2003 Physiol. Meas. 24413

[9] Smith D R, Pendry J B and Wiltshire M C K 2004 Science 305788

[10] Engheta N and Ziolkowski R W 2006 Electromagnetic Metamaterials: Physics and Engineering Explorations (New York: Wiley)

[11] Solymar L and Shamonina E 2009 Waves in Metamaterials (Oxford: Oxford University Press)

[12] Marques R, Martin F and Sorolla M 2008 Metamaterials with Negative Parameters: Theory, Design and Microwave Applications (Hoboken, NJ: Wiley)

[13] Sarychev A K and Shalaev V M 2007 Electrodynamics of Metamaterials (Singapore: World Scientific)

[14] Schurig D, Mock J J, Justice B J, Cummer S A, Pendry J B, Starr A F and Smith D R 2006 Science 314977

[15] Kundtz N B, Gaultney D and Smith D R 2010 New J. Phys. 12043039

[16] Leonhardt U 2006 Science 312 1777-80

[17] Nachman A I 1988 Ann. Math. 128531

[18] Wolf E and Habashy T 1993 J. Mod. Opt. 40785

[19] Cai W, Chettiar U K, Kildishev A V, Shalaev V M and Milton G W 2007 Appl. Phys. Lett. 91111105

[20] Kildishev A V, Cai W, Chettiar U K and Shalaev V M 2008 New J. Phys. 10115029

[21] Cummer S A, Popa B-I, Schurig D, Smith D R and Pendry J 2006 Phys. Rev. E 74036621

[22] Cai W, Chettiar U K, Kildishev A V and Shalaev V M 2007 Nat. Photon. Lett. 1224

[23] Cummer S A, Liu R and Cui T J 2009 J. Appl. Phys. 105056102

[24] Urzhumov Y A, Ghezzo F, Hunt J and Smith D R 2010 New J. Phys. 12073014

[25] Blanchard C, Wu B-I, Porti J A, Chen H, Zhang B and Morente J A 2009 J. Opt. Soc. Am. B 262117

[26] Yan M, Ruan Z and Qiu M 2007 Phys. Rev. Lett. 99233901

[27] van de Hulst H C 1957 Light Scattering by Small Particles (New York: Wiley)

[28] Popa B-I and Cummer S A 2009 Phys. Rev. A 79023806

[29] Parazzoli C G, Koltenbah B E C, Greegor R B, Lam T A and Tanielian M H 2006 J. Opt. Soc. Am. B 23439

[30] Schurig D, Pendry J B and Smith D R 2006 Opt. Express 149794

[31] Leonhardt U 2006 New J. Phys. 8118

[32] Li J and Pendry J B 2008 Phys. Rev. Lett. 101203901

[33] Kundtz N B 2009 Advances in complex artificial electromagnetic media $P h D$ Thesis Duke University, Durham, NC

[34] Liu R, Ji C, Mock J J, Chin J Y, Cui T J and Smith D R 2009 Science 323366

[35] Landau L D and Lifshitz E M 1976 Mechanics 3rd edn (Oxford: Elsevier/Butterworth-Heinemann) Problem 2 